Article

\title{
Ge-Core/a-Si-Shell Nanowire-Based Field-Effect Transistor for Sensitive Terahertz Detection
}

\author{
Xiangying Deng ${ }^{1}$ (D), Marolop Simanullang ${ }^{\text {(iD) }}$ and Yukio Kawano ${ }^{1, *}$ \\ 1 Laboratory for Future Interdisciplinary Research of Science and Technology, \\ Department of Electrical and Electronic Engineering, Tokyo Institute of Technology 2-12-1, Ookayama, \\ Meguro-ku, Tokyo 152-8552, Japan; deng.x.ab@m.titech.ac.jp \\ 2 Department of Electrical Engineering, Faculty of Science and Technology, Tokyo University of Science, \\ 2641 Yamazaki, Noda-shi, Chiba 278-8510, Japan; marolop@rs.tus.ac.jp \\ * Correspondence: kawano@pe.titech.ac.jp; Tel.: +81-03-5734-2542
}

Received: 19 March 2018; Accepted: 27 May 2018; Published: 29 May 2018

\begin{abstract}
Although terahertz technology has demonstrated strong potential for various applications, detectors operating in the terahertz region are yet to be fully established. Numerous designs have been proposed for sensitive terahertz detection, with a nanowire-based field-effect transistor (FET) being one of the most promising candidates. In this study, we use a Ge-core/a-Si-shell nanowire coupled to a bow-tie antenna to fabricate a FET structure for terahertz detection. We achieved high responsivity and low noise equivalent power (NEP) upon irradiation at $1.63 \mathrm{THz}$. The proposed sensitive terahertz detector will further promote the development of terahertz technology in fields such as spectroscopic analysis and imaging.
\end{abstract}

Keywords: terahertz; nanowire; photodetector; planar antenna; field-effect transistor

\section{Introduction}

The terahertz spectrum is referred to as the terahertz gap and is defined as the frequency range from $0.1 \mathrm{THz}$ to $10 \mathrm{THz}$. This frequency region is located between the infrared and microwave regions; it has attracted many researchers and has been studied since the late nineteenth century.

Terahertz technology shows advantages such as characteristic absorption bands for many materials and molecules, high atmospheric absorption, and a nondestructive imaging capability. These advantages have enabled the terahertz ranges to stand out from all other frequency ranges for electromagnetic waves, promising terahertz technologies with strong potential in various fields such as biochemical and medical sensing, information and communication systems, semiconductor and superconductor characterization, non-destructive evaluation, quality control of food and agricultural products, and space and defense industries [1-9].

Although the terahertz spectrum promises many significant advantages, obstacles still exist in the evolution of terahertz technology. For instance, current terahertz power generation and receiver technologies are inefficient and impractical. These barriers hamper utilization of the terahertz region compared to neighboring bands because of well-developed optical engineering of low-frequency infrared detectors and already mature technologies for the highest frequencies of the radio spectrum in the microwave region. One of the development limitations is that the frequencies of terahertz waves are too high for current high-frequency electronics. General detectors for high-frequency electronics are mostly based on various high-speed transistors and complicated oscillating circuits, which are limited in operation to approximately hundreds of GHz. Another limitation is that the photon energy of a terahertz wave is much lower than that of visible light, with devices based on traditional optoelectronic materials being extendable only to tens of $\mathrm{THz}[10,11]$. 
Following the development of higher-power sources and nanotechnology for sensitive detectors, terahertz technology has been pushed to the forefront. Numerous breakthroughs have greatly improved the performance of terahertz systems. Details of historical achievements and the fundamental principles of terahertz research can be found in several reviews and books [8,9]. The unique advantages and expected applications of the terahertz spectrum have motivated the fabrication of terahertz detectors based on various technologies. Existing technologies include bolometers, thermoelectric sensors, Schottky barrier diodes, quantum effect sensors, and nanowire photoconductive detectors [12-14]. However, these techniques have shortcomings, including high cost and complexity, low detection rate, and lack of sensitivity, which affect their usefulness in practical applications. Thermal sensing elements that detect terahertz radiation as heat are either very slow in modulation frequency $\left(10^{2} \sim 10^{3} \mathrm{~Hz}\right)$ or require a harsh operating environment such as deep cryogenic cooling to $\sim 4 \mathrm{~K}$ for superconducting hot-electron bolometers [15]. The terahertz time domain spectroscopy (THz-TDS) system is based on photoconductive detection and electro-optical detection, which works through detection of a terahertz light-induced change in the refractive index of the electro-optical materials. The THz power detected in the THz-TDS is about $1 \mu \mathrm{W}$, limiting application in real sample spectroscopy and imaging [16,17]. Moreover, data can only be acquired one pixel at a time, which limits the data acquisition rate and largely slows the imaging time. Therefore, the development of technologies for high sensitivity, high responsivity, and low noise equivalent power (NEP) terahertz detectors is strongly demanded for various promising applications. Recently, nanomaterials, together with nanotechnology, have come to challenge many of these requirements and have been widely employed in the design and fabrication of terahertz detectors [18,19].

Recently, complementary metal oxide semiconductor (CMOS) and the field-effect transistor (FET) [20] have been actively studied as terahertz detectors. The detection mechanism for such devices is based on the excitation of an electromagnetic field in the transistor channel [21]. The operating conditions include the following: a plasma damping rate lower than the inverse of the plasmon transit time in the transistor channel, together with a plasma damping rate lower than the frequency of the incident terahertz signal [22]. When the impinging electromagnetic radiation contains frequencies higher than $1 \mathrm{THz}$, these conditions result in a mobility value greater than $104 \mathrm{~cm}^{2} /(\mathrm{V} \cdot \mathrm{s})$ for the electrons in the transistor channel. However, when the plasma oscillations are over damped, the detector can function with a broadband detection mechanism. In this situation, the electromagnetic radiation induces an oscillating variation in the FET gate voltage. The gate voltage then biases the channel, modulating the carrier density and the drift velocity, thereby affecting the electron dynamics. Thus, the incident signal can be detected as a DC voltage across the channel under open-circuit conditions. This detection mechanism offers advantages such as sensitive detection and fast response times. This device structure is also convenient for application to large arrays. For example, CMOS and 2D FETs utilizing these schemes have been demonstrated to be functional at high frequencies [23]. FETs based on nanowires (NWs) as the channel have shown promise for room-temperature, high-sensitivity terahertz detection $[18,19]$. In such devices, the impinging signal is collected by a resonant or a broadband antenna and then fed in to the source and gate terminals. Table 1 lists the responsivity and NEP from recent studies of NW-based FETs that operate in the terahertz spectral region [24-26].

Table 1. Responsivity and noise equivalent power (NEP) from recent studies on NW-based FETs.

\begin{tabular}{ccc}
\hline Research Group & $\boldsymbol{R}_{\boldsymbol{v}}(\mathrm{V} / \mathbf{W})$ & NEP $(\mathbf{W} / \sqrt{ } \mathbf{H z})$ \\
\hline Romeo et al. [24] & $0.9-6.0$ & $\sim 10^{-9}$ \\
Pitanti et al. [25] & 1.5 & $2.3 \times 10^{-9}$ \\
Viti et al. [26] & 1.0 & $\sim 10^{-8}$ \\
\hline
\end{tabular}

In this paper, we present our NW-based FET terahertz detector. We use Ge-core/amorphous-Si (a-Si)-shell NWs as the active material for the FET detector and employ a bow-tie antenna as the 
metallic planar antenna for radiation coupling. The Ge-core/a-Si-shell NWs exhibits a much higher field-effect hole mobility compared to the Ge NWs without a-Si-shell, which may lead to a better performance of the device. Electronic characteristics and photoresponse measurements are also carried out in this work. High responsivity with low NEP was demonstrated, which is superior to the results of related studies.

\section{Materials and Methods}

We have implemented a FET based on Ge-core/a-Si-shell NWs. The hole mobility for Ge is higher than those of Si and III-V semiconductors. Ge is suitable for use as the channel material for high-speed $p$-channel FETs [27]. However, the pure Ge material is easily oxidized. The native oxide of Ge is unstable and soluble in water, making preservation of the Ge NW difficult, which in turn affects the durability of a Ge NW-based device. Therefore, additional artificial surface passivation of Ge NWs is required. Organic layers such as alkanethiols [28], aqueous halide solutions [29], and dielectric layers such as aluminum oxide $\left(\mathrm{Al}_{2} \mathrm{O}_{3}\right)$ and hafnium(IV) oxide $\left(\mathrm{HfO}_{2}\right)$ [30] can be used to passivate the Ge NW surface. The current method for surface passivation includes use of an a-Si shell deposited by chemical vapor deposition (CVD). Figure 1 shows a Ge-core/a-Si-shell NW fabricated using CVD, which is used for device fabrication in this work. Our Ge NW cores were grown epitaxially on Ge (111) substrate. High-resolution TEM shows that an individual Ge NW core is a single crystal with a diamond structure and a preferred growth along the $<111\rangle$ direction. The lattice fringe spacing is measured to be $\sim 0.32 \mathrm{~nm}$. The defect density of the germanium core was around $0.2 / \mu \mathrm{m}$. The amorphous silicon shell was deposited conformally along the observed length. The average diameter of the germanium nanowire core was $\sim 28 \mathrm{~nm}$, the amorphous silicon shell thickness was $\sim 5 \mathrm{~nm}$, and the average of the nanowire length was $\sim 1.2 \mu \mathrm{m}$. We selected those nanowires with thinner diameter and longer length for further device fabrication. The field-effect hole mobility of Ge-core/Si-shell NWs used in this work is $664 \mathrm{~cm}^{2} / \mathrm{Vs}$, measured at $\left|V_{\mathrm{sd}}\right|=0.1 \mathrm{~V}$. Compared to the typical field-effect hole mobility in Ge NWs (without an a-Si-shell) of $213 \mathrm{~cm}^{2} / \mathrm{Vs}$ [27], the Ge-core/Si-shell NWs promises a better performance.
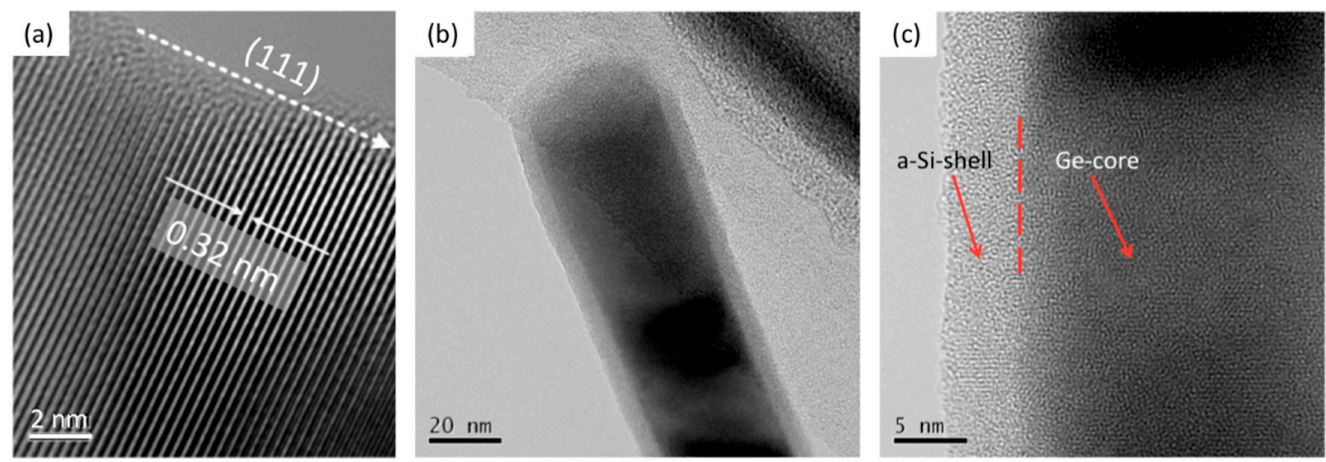

Figure 1. TEM image of the Ge-core/a-Si-shell NW. (a) Crystal structure. (b) One end of the nanowire. (c) Magnified image of the intersection of the Ge-core and a-Si-shell, showing an excellent interface between the Ge-core and a-Si shell.

A bow-tie antenna was chosen for coupling with the Ge-core-/a-Si-shell NW. The bow-tie antenna is a common antenna that has been frequently used for NW-based terahertz detectors. It comprises two axisymmetric-placed sectors. The bow-tie antenna is a wide-band antenna, but its frequency range and bandwidth are determined by the radius $r$ of the sectors. The relationship between $r$ and $f$ is given by:

$$
\frac{c}{r}=f
$$

$c$ is the speed of light, and $f$ is the designed frequency of the antenna. 
In this study, we used terahertz gas lasers pumped by $\mathrm{CO}_{2}$ infrared lasers as the terahertz source, whose effective frequency was $1.63 \mathrm{THz}$. In this case, we designed the radius of our bow-tie antenna to be $200 \mu \mathrm{m}$. A side-gate geometry was used for the device structure. The designed distance between the gate and the nanowire was $200 \mathrm{~nm}$. The source and gate electrodes were connected to the two lobes of the bow-tie antenna, respectively, whereas the drain electrode was connected directly to the bonding pad. The three bonding pads were connected to a power source, a current amplifier, and a lock-in amplifier, as depicted in Figure 2. In the nanowire-based field effect transistors, the conductivity of the nanowire can be controlled through an external field generated by a gate electrode. The two halves of the bow-tie antenna connected to the gate and source electrodes create an inherent asymmetry between the source and drain. An ac potential is induced by the incoming radiation between the gate and source electrodes. The modulation of the carrier density of the nanowire and their drift velocity results in the rectification of the $a c$ potential, which in turn leads to a $d c$ photoresponse between source and drain.

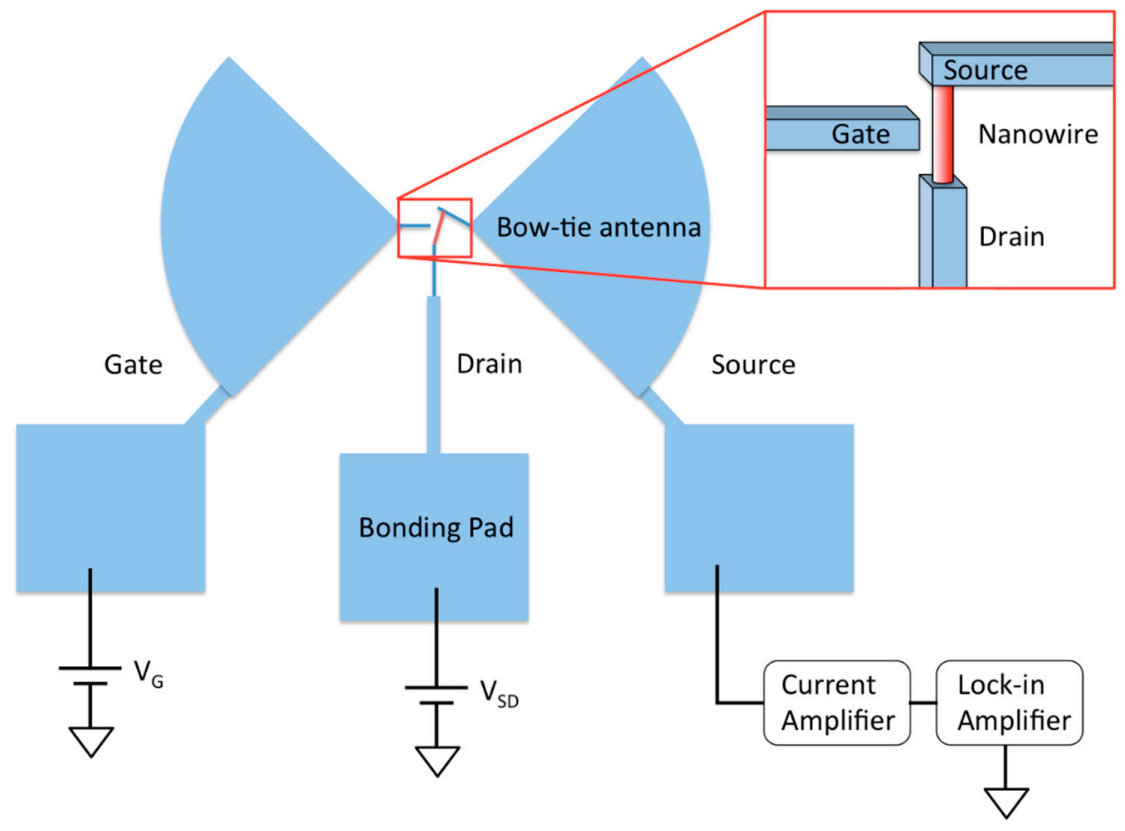

Figure 2. Illustration showing the device structure for an NW-based FET coupled to a bow-tie antenna. The bow-tie antenna is designed with a resonance frequency range of approximately $1.63 \mathrm{THz}$. A Ge-core/a-Si-shell NW is connected to the bow-tie antenna through electrodes (inset). The bonding pads are designed, along with the device, for on-chip measurement in a cryogenic vacuum chamber. The electrical connections of the detection setup are indicated in the figure.

The device was fabricated according to the following procedure. A Si wafer was cut into $2 \mathrm{~cm} \times 2 \mathrm{~cm}$ squares. The cleaned $\mathrm{Si}$ substrate was then placed into an oxidation oven for formation of the $\mathrm{SiO}_{2}$ layer. In this device, the thickness of the $\mathrm{SiO}_{2}$ layer was designed to be $100 \mathrm{~nm}$. The Ge-core/a-Si-shell NWs were initially grown onto a Ge wafer. To remove the NWs from the substrate, we submerged the wafer into IPA and then ultrasonically agitated it. The NWs were then suspended inside the IPA liquid. Next, we used a dropper to drop cast the NW liquid onto our substrate. During drop casting, frequent checking under a microscope is recommended to ensure that an appropriate number of NWs are on the substrate. The substrate was then transferred to a scanning electron microscope to locate the NWs and record micrographs. Figure 3 shows a micrograph of a NW lying next to an address mark. 


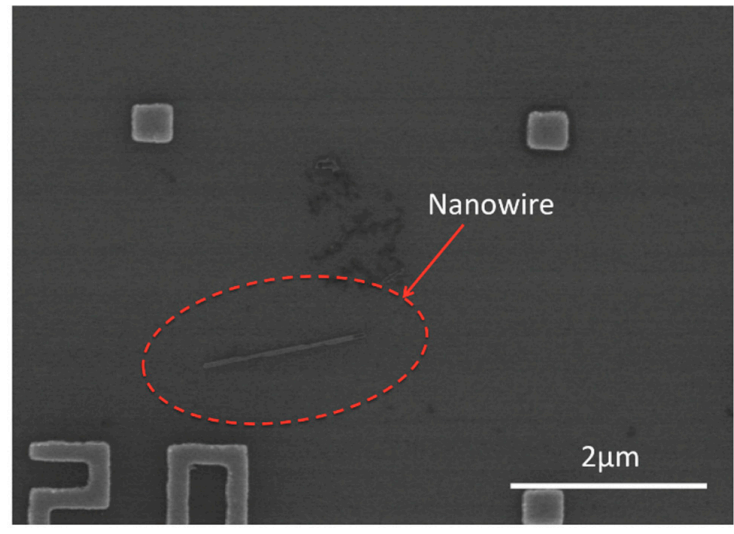

Figure 3. Ge-core/a-Si-shell NW observed by SEM. The NW is in the middle part of the micrograph. Metal dots and numbers around the NWs are address marks used to locate the NW and design electrodes.

To ensure a good ohmic contact between the source/drain electrodes and the NW, the oxidation layer on the surface of the NW should be removed. The sample is submerged in BHF for $5 \mathrm{~s}$ and rinsed in water before being placed into an EBE for metal deposition. Previous experiments have shown that $\mathrm{Ni}$, in comparison with other transition metals, can form a better alloy with the core-shell NWs, which is expected to lead to a better ohmic contact. Therefore, $\mathrm{Ni} / \mathrm{Au}$ was used as the electrode material as well as contact material for future bonding. In our experiments, the thickness for the deposited Ni was $50 \mathrm{~nm}$ and the thickness for Au deposition was $70 \mathrm{~nm}$. After EBE deposition, the substrate was submerged in acetone for lift-off, rinsed in IPA and water, and then prepared for measurement. Figure 4 presents SEM micrographs of the fabricated device.

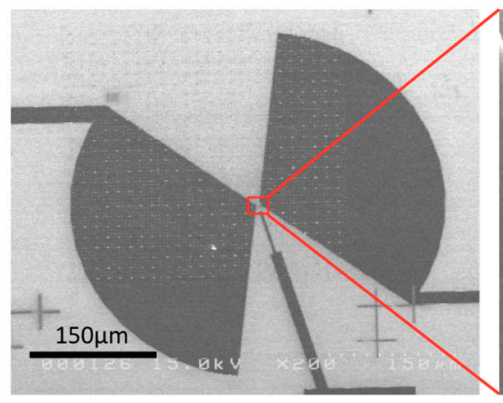

(a)

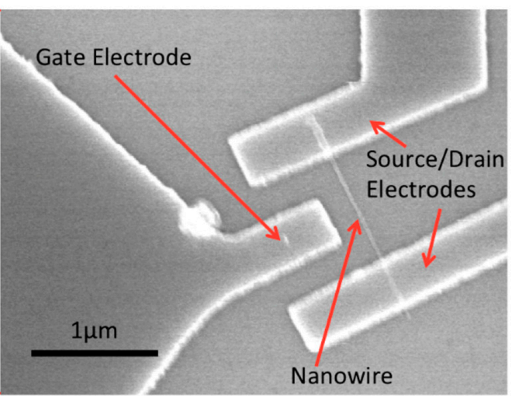

(b)

Figure 4. SEM image of the fabricated NW-based FET. (a) General view of the NW-based FET with a bow-tie antenna terahertz detector. The two circular sectors are the bow-tie antenna, with electrodes connecting outward to the bonding pads. (b) Enlarged view around the NW. Thinner electrodes are used around the NWs for the source, drain, and side-gate electrodes. The NW lies between the source and drain electrodes, at a fixed distance from the side-gate electrode.

\section{Measurement Results and Discussion}

The electronic characteristics of the fabricated devices were measured using a probe station. The results show ohmic contact between the source/drain electrodes and the NW, along with good gate modulation via the side-gate, as shown in Figure 5. The source-drain current was reduced from $10^{-8} \mathrm{~A}$ to $10^{-12} \mathrm{~A}$ when the gate voltage was increased from $10 \mathrm{~V}$ to $14 \mathrm{~V}$. The gate leakage and noise value during the measurements were both $10^{-13} \mathrm{~A}$, which indicates a good measurement environment with negligible noise. This gate modulation indicates the potential for terahertz detection starting at a gate voltage of $10 \mathrm{~V}$. 


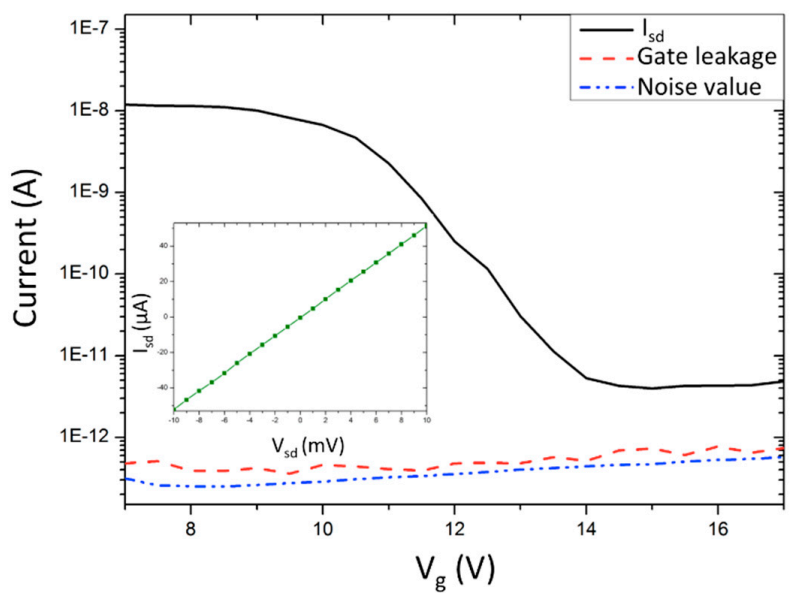

Figure 5. Gate modulation and $I-V$ characteristics (inset) of a NW measured on the probe stage. The black line indicates the source-drain current during the measurement. Red and blue dashes represent the side-gate leakage and noise value during the measurement, respectively. The inset figure shows the $I-V$ characteristics for the Ge-core/a-Si shell NW.

The devices were subsequently cut and bonded to chip carriers using an ultrasonic bonding machine. The bonding wire was Al. The bonded chips were transferred to the cryogenic vacuum chamber for measurement of their terahertz response. The pressure inside the chamber was maintained at less than $10^{-5} \mathrm{~Pa}$, and the temperature inside the chamber was fixed at $4 \mathrm{~K}$. We used a terahertz gas laser pumped by $\mathrm{CO}_{2}$ infrared lasers as the terahertz source, with the frequency set to approximately $1.63 \mathrm{THz}$. The terahertz irradiation was guided through an optical fiber and then transmitted through the transparent aperture into the cryogenic vacuum chamber. The intensity was mechanically chopped, and the induced $d c$ photo response was read out at the source electrode by a lock-in amplifier. Upon application of the terahertz signal, a terahertz response was measured as a function of drain voltage; the results are shown in Figure 6a. The drain voltage was swept from $-10 \mathrm{mV}$ to $10 \mathrm{mV}$ with the gate voltage fixed at $11 \mathrm{~V}$ and $13 \mathrm{~V}$ respectively.

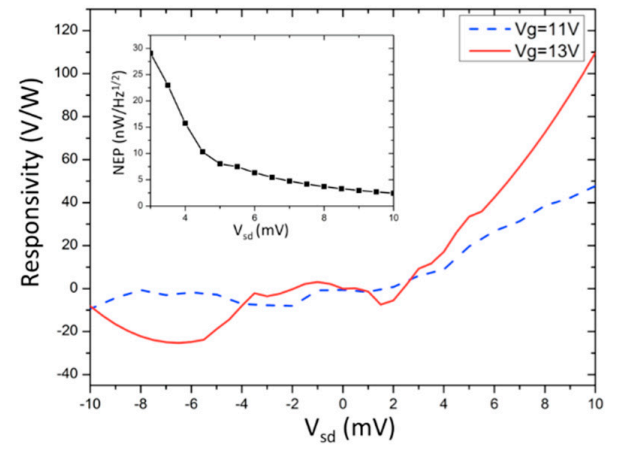

(a)

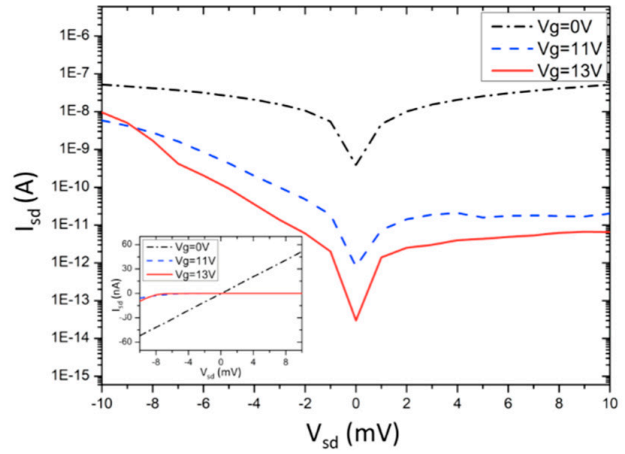

(b)

Figure 6. Measurement results of the fabricated device in the cryogenic vacuum chamber. (a) Terahertz responsivity for the NW-based FET terahertz detector under $1.63 \mathrm{THz}$ in a cryogenic vacuum chamber. Terahertz photoresponse versus the drain voltage were displayed at gate voltage of $11 \mathrm{~V}$ and $13 \mathrm{~V}$ respectively. The NEP at gate voltage of $13 \mathrm{~V}$ (inset) was estimated based on the data for the gate voltage of $13 \mathrm{~V}$. (b) $I-V$ characteristic of the NW-based FET device on a log-scale when the gate voltage was $0 \mathrm{~V}, 11 \mathrm{~V}$, and $13 \mathrm{~V}$ respectively. The inset is the linear-scale of $I-V$ characteristic.

The $I-V$ characteristic of the NW-based FET (Figure $6 b$ ) was asymmetric when the gate voltage of $11 \mathrm{~V}$ and $13 \mathrm{~V}$ were applied to the device, which leads to the asymmetric $\mathrm{THz}$ photoresponse of 
the device with respect to the source-drain voltage. Moreover, the measurement results exhibit higher responsivity under higher gate voltage $(13 \mathrm{~V})$ when the source-drain current $I_{s d}$ is nearly vanishing. Such a behavior can be explained using a simple diffusive model: [31,32]

$$
\Delta u \propto \frac{1}{\sigma} \frac{d \sigma}{d V_{g}}
$$

where $\sigma$ is the conductivity of the FET, $\Delta u$ is the induced dc photoresponse. The formula indicates that the photoresponse is proportional to the derivative of the conductivity as a function of gate voltage, and inversely proportional to the conductivity of the FET. Considering that the value of $d \sigma / d V_{g}$ is similar for $V_{g}=11 \mathrm{~V}$ and $V_{g}=13 \mathrm{~V}$, the dramatical decrease of FET conductivity $\sigma$ as the gate voltage change from $11 \mathrm{~V}$ to $13 \mathrm{~V}$ results in an increase of the responsivity. The calculated ratio between the photoresponse for $V_{g}=13 \mathrm{~V}$ and $V_{g}=11 \mathrm{~V}$ is:

$$
\frac{\Delta u_{V_{g}=13 \mathrm{~V}}}{\Delta u_{V_{g}=11 \mathrm{~V}}}=\frac{3.377}{1.694} \approx 2
$$

which is similar to our experimental value:

$$
\frac{R_{v} V_{g}=13 \mathrm{~V}}{R_{v V_{g}}=11 \mathrm{~V}}=\frac{109.6 \mathrm{~V} / \mathrm{W}}{47.7 \mathrm{~V} / \mathrm{W}} \approx 2.3
$$

Additionally, the data shows a larger photoresponse at higher drain voltages, confirming the photovoltage effect of the measurement results. When gate voltage of $13 \mathrm{~V}$ is applied, the photocurrent in the NW-based FET reached $56.7 \mathrm{nA}$ upon terahertz irradiation at $1.63 \mathrm{THz}$ at a drain voltage of $10 \mathrm{mV}$. The power of the irradiated CW THz beam was $0.5 \mathrm{~mW}$, measured at the output of the optic fiber. Considering the diameter of $\mathrm{THz}$ spot size was $0.9 \mathrm{~mm}$ while the diameter of our bow-tie antenna was $0.4 \mathrm{~mm}$, we estimated the effective power illuminated into the antenna area was $0.1 \mathrm{~mW}$. This leads to a responsivity of approximately $109.6 \mathrm{~V} / \mathrm{W}$. The NEP is estimated from the ratio $N / R_{v}[25,33]$. $R_{v}$ is the THz-detected voltage signal, divided by the effective $\mathrm{THz}$ power illuminated onto the antenna of the detector. The Johnson-Nyquist noise is expressed as $N=\sqrt{ }\left(4 k_{B} R_{n w} T\right)$, which gives $2.69 \times 10^{-7} \mathrm{~V} / \sqrt{ } \mathrm{Hz}$ for our device. Here $k_{B}$ is the Boltzmann constant, $R_{n w}$ is the resistance of the nanowire, and $T$ is the measurement temperature. The shot noise is expressed as $N=\sqrt{ }\left(2 e i_{d c}\right)$, which gives $3.28 \times 10^{-16} \mathrm{~A} / \sqrt{ } \mathrm{Hz}\left(1.05 \times 10^{-8} \mathrm{~V} / \sqrt{ } \mathrm{Hz}\right)$ for our device. Here $e$ is the elementary charge, and $i_{d c}$ is the average $d c$ current. The Johnson-Nyquist noise is thus the main source of noise in our measurement. Based on this, we extracted the NEP of the Ge-core/a-Si-shell NW-based FET terahertz detector, as shown in the inset in Figure 6. A steady NEP appeared when the source-drain voltage was greater than $4 \mathrm{mV}$. A minimum NEP value of $6.29 \times 10^{-10} \mathrm{~W} / \mathrm{Hz}^{1 / 2}$ was achieved at $V_{g}=11 \mathrm{~V}$ by our fabricated Ge-core/a-Si-shell NW-based THz detector. If the coupling efficiency of the bow-tie shaped antenna is roughly $80 \%$ [34], the intrinsic NEP value is estimated to be $5.03 \times 10^{-10} \mathrm{~W} / \mathrm{Hz}^{1 / 2}$.

The improved performance of our Ge-core/a-Si-shell NW-based FET detector is mainly due to high field-effect hole mobility of the Ge-core NW protected by the surrounding a-Si-shell. We selected the NWs with thinner diameter to ensure higher carrier density. The well matching of impedance between the NW-based FET and the bow-tie antenna also led to a better performance. Compared with other recent related studies [20-22], the detectivity of our Ge-core/a-Si-shell NW-based FET terahertz detector is superior, along with a smaller NEP. These features indicate that the Ge-core/a-Si-shell NWs show promise as a valid alternative to conventional InAs materials for terahertz detection, with increased sensitivity and a lower NEP. The current distance between the gate and the nanowire was $200 \mathrm{~nm}$. The reduction in this distance will result in the responsivity enhancement. However, the current side-gated model has limitations during fabrication, and it is difficult to achieve a shorter gate-nanowire distance. Top-gated or back-gated model is a possible method to further reduce the gate-nanowire distance and improve the responsivity. 


\section{Conclusions}

We used a Ge-core/a-Si-shell NW as the channel in a field-effect transistor (FET) considering the high hole mobility of Ge. A Si shell was used to passivate the surface of the Ge to protect it from oxidation and extend its preservation time. The NW was coupled with a bow-tie antenna, which could concentrate and transform the incident terahertz irradiation into an electric field to modulate the channel. An improved responsivity of $109.6 \mathrm{~V} / \mathrm{W}$ and a minimum NEP of $6.29 \times 10^{-10} \mathrm{~W} / \mathrm{Hz}^{1 / 2}$ was detected at a frequency of $1.63 \mathrm{THz}$, which is mainly due to the high field-effect hole mobility in the Ge-core/a-Si-shell NWs. Such a photoresponse demonstrated sensitive detection in the terahertz region. The presented Ge-core/a-Si-shell NW-based detector will contribute to sensitive detection in the terahertz spectral range and will further promote the development of terahertz technology.

Author Contributions: X.D. designed, fabricated, and conducted the electronic characteristic measurement of the device; M.S. synthesized the nanowire and analyzed the material properties; X.D. and Y.K. analyzed the data; X.D. wrote the paper. All authors read and commented on the manuscript.

Funding: This work was supported in part by the JST-Mirai Program, the Matching Planner Program, and the Center of Innovation Program from the Japan Science and Technology Agency, (JSPS KAKENHI Grant Numbers 18H03766, JP17K19026, JP17H02730, JP16H00798, JP16H00906, JP16J09937) from the Japan Society for the Promotion of Science, the Murata Science Foundation, and Support for Tokyo tech Advanced Researchers (STAR).

Conflicts of Interest: The authors declare no conflict of interest. The founding sponsors had no role in the design of the study; in the collection, analyses, or interpretation of data; in the writing of the manuscript; or in the decision to publish the results.

\section{References}

1. Clery, D. Brainstorming their way to an imaging revolution. Science 2002, 297, 761-763. [CrossRef] [PubMed]

2. Zeitler, J.A.; Shen, Y.C. Industrial applications of terahertz imaging. Terahertz Spectrosc. Imaging 2012, 171, 451-489. [CrossRef]

3. Nakajima, S.; Hoshina, H.; Yamashita, M.; Otani, C. Terahertz imaging diagnostics of cancer tissues with a chemometrics technique. Appl. Phys. Lett. 2007, 90, 041102. [CrossRef]

4. Kleine-Ostmann, T.; Nagatsuma, T. A review on terahertz communications research. J. Infrared Millim. Terahertz Waves 2011, 32, 143-171. [CrossRef]

5. Brun, M.A.; Formanek, F.; Yasuda, A.; Sekine, M.; Ando, N.; Eishii, Y. Terahertz imaging applied to cancer diagnosis. Phys. Med. Biol. 2010, 55, 4615-4623. [CrossRef] [PubMed]

6. Ariyoshi, S.; Otani, C.; Dobroiu, A.; Sato, H.; Kawase, K.; Shimizu, H.M. Terahertz imaging with a direct detector based on superconducting tunnel junctions. Appl. Phys. Lett. 2006, 88, 203503. [CrossRef]

7. Kulesa, C. Terahertz spectroscopy for astronomy: From comets to cosmology. IEEE Trans. Terahertz Sci. Technol. 2011, 1, 232-240. [CrossRef]

8. Tonouchi, M. Cutting-edge terahertz technology. Nat. Photonics 2007, 1,97-105. [CrossRef]

9. Kawano, Y. Terahertz waves: A tool for condensed matter, the life sciences and astronomy. Contemp. Phys. 2013, 54, 143-165. [CrossRef]

10. Kleiner, R. Filling the terahertz gap. Science 2007, 23, 1254-1255. [CrossRef] [PubMed]

11. Sirtori, C. Applied physics: Bridge for the terahertz gap. Nature 2002, 417, 132-133. [CrossRef] [PubMed]

12. Peng, K.; Parkinson, P.; Boland, J.; Gao, Q.; Wenas, Y.; Davies, C.; Li, Z.; Fu, L.; Johnston, M.; Tan, H.; et al. Broadband phase-sensitive single InP nanowire photoconductive terahertz detectors. Nano Lett. 2016, 16, 4925-4931. [CrossRef] [PubMed]

13. Peng, K.; Parkinson, P.; Fu, L.; Gao, Q.; Jiang, N.; Guo, Y.; Wang, F.; Joyce, H.; Boland, J.; Tan, H.; et al. Single nanowire photoconductive terahertz detectors. Nano Lett. 2015, 15, 206-210. [CrossRef] [PubMed]

14. Otsuji, T. Trends in the research of modern terahertz detectors: Plasmon detectors. IEEE Trans. Terahertz Sci. Technol. 2015, 5, 1110-1120.

15. Sizov, F.; Rogalski, A. THz detectors. Prog. Quantum Electron. 2010, 34, 278-347. [CrossRef]

16. Hangyo, M.; Tani, M.; Nagashima, T. Terahertz time-domain spectroscopy of solids: A review. Int. J. Infrared Millim. Waves 2005, 26, 1661-1690. [CrossRef] 
17. Meng, F.; Thomson, M.; Molter, D.; Loffler, T.; Jonuscheit, J.; Beigang, R.; Bartschke, J.; Bauer, T.; Nittmann, M.; Roskos, H. Coherent electro-optical detection of terahertz radiation from an optical parametric oscillator. Opt. Express 2010, 18, 11316-11326. [CrossRef] [PubMed]

18. Vitiello, M.S.; Viti, L.; Romeo, L.; Ercolani, D.; Scalari, G.; Faist, J.; Beltram, F.; Sorba, L.; Tredicucci, A. Semiconductor nanowires for highly sensitive, room-temperature detection of terahertz quantum cascade laser emission. Appl. Phys. Lett. 2012, 100, 241101. [CrossRef]

19. Vitiello, M.S.; Coquillat, D.; Viti, L.; Ercolani, D.; Teppe, F.; Pitanti, A.; Beltram, F.; Sorba, L.; Knap, W.; Tredicucci, A. Room-temperature terahertz detectors based on semiconductor nanowire field-effect transistors. Nano Lett. 2012, 12, 96-101. [CrossRef] [PubMed]

20. Knap, W.; Teppe, F.; Meziani, Y.; Dyakonova, N.; Lusakowski, J. Plasma wave detection of sub-terahertz and terahertz radiation by silicon field-effect transistors. Appl. Phys. Lett. 2004, 85, 675. [CrossRef]

21. Dyakonov, M.; Shur, M. Detection, mixing, and frequency multiplication of terahertz radiation by two-dimensional electronic fluid. IEEE Trans. Electron Devices 1996, 43, 380-387. [CrossRef]

22. Teppe, F.; Knap, W. Room-temperature plasma waves resonant detection of sub-terahertz radiation by nanometer field-effect transistor. Appl. Phys. Lett. 2005, 87, 052107. [CrossRef]

23. Nadar, S.; Videlier, H.; Coquillat, D.; Teppe, F.; Sakowicz, M.; Dyakonova, N.; Knap, W.; Seliuta, D.; Kasalynas, I.; Valusis, G. Room temperature imaging at 1.63 and $2.54 \mathrm{THz}$ with field effect transistor detectors. J. Appl. Phys. 2010, 108, 054508. [CrossRef]

24. Romeo, L.; Coquillat, D.; Pea, M.; Ercolani, D.; Beltram, F.; Sorba, L.; Knap, W.; Tredicucci, A.; Vitiello, M.S. Nanowire-based field effect transistors for terahertz detection and imaging systems. Nanotechology 2013, 24, 214005. [CrossRef] [PubMed]

25. Viti, L.; Coquillat, D.; Ercolani, D.; Knap, W.; Sorba, L.; Vitiello, M.S. High-performance room-temperature $\mathrm{THz}$ nanodetectors with a narrowband antenna. In Proceedings of the Terahertz, RF, Millimeter, and Submillimeter-Wave Technology and Applications VII, San Francisco, CA, USA, 2 April 2014. [CrossRef]

26. Pitanti, A.; Vitiello, M.S.; Romeo, L.; Coquillat, D.; Teppe, F.; Knap, W.; Ercolani, D.; Sorba, L.; Tredicucci, A. Semiconductor nanowire field-effect transistors: Towards high-frequency THz detectors. Proc. SPIE 2012, 8496. [CrossRef]

27. Simanullang, M.; Wisna, G.B.M.; Usami, K.; Cao, W.; Kawano, Y.; Banerjee, K.; Oda, S. Undoped and catalyst-free germanium nanowires for high-performance p-type enhancement-mode field-effect transistors. J. Mater. Chem. C 2016, 4, 5102-5108. [CrossRef]

28. Wang, D.; Dai, H. Germanium nanowires: From synthesis surface chemistry, and assembly to devices. Appl. Phys. A Mater. Sci. Process. 2006, 85, 217-225. [CrossRef]

29. Jagannathan, H.; Kim, J.; Deal, M.; Kelly, M.; Nishi, Y. Halide passivation of germanium nanoiwres. ECS Trans. 2006, 3, 1175-1180. [CrossRef]

30. Simanullang, M.; Usami, K.; Noguchi, T.; Surawijaya, A.; Kodera, T.; Kawano, Y.; Oda, S. Surface passivation of germanium nanowires using $\mathrm{Al}_{2} \mathrm{O}_{3}$ and $\mathrm{HfO}_{2}$ deposited via atomic layer deposition technique. Jpn. J. Appl. Phys. 2014, 53, 06JG04-1-06JG04-6. [CrossRef]

31. Knap, W.; Kachorovskii, V.; Deng, Y.; Rumyantsev, S.; Lu, J.; Gaska, R.; Shur, M.; Simin, G.; Hu, X.; Khan, M.; et al. Nonresonant detection of terahertz radiation in field effect transistors. J. Appl. Phys. 2002, 91, 9346. [CrossRef]

32. Lisauskas, A.; Pfeiffer, U.; Ojefors, E.; Bolivar, P.; Glaab, D.; Roskos, H. Rational design of high-responsivity detectors of terahertz radiation based on distributed self-mixing in silicon field-effect transistors. J. Appl. Phys. 2009, 105, 114511. [CrossRef]

33. Vicarelli, L.; Vitiello, M.S.; Coquillat, D.; Lombardo, A.; Ferrari, A.C.; Knap, W.; Polini, M.; Pellegrini, V.; Tredicucci, A. Graphene field-effect transistors as room-temperature terahertz detectors. Nat. Mater. 2012, 11, 865-871. [CrossRef] [PubMed]

34. Bauer, M.; Ramer, A.; Boppel, S.; Chevtchenko, S.; Lisauskas, A.; Heinrich, W.; Krozer, V.; Roskos, H.G. Highsensitivity wideband THz detectors based on GaN HEMTs with integrated bow-tie antennas. In Proceedings of the 10th European Microwave Integrated Circuits Conference, Paris, France, 7-8 September 2015.

(C) 2018 by the authors. Licensee MDPI, Basel, Switzerland. This article is an open access article distributed under the terms and conditions of the Creative Commons Attribution (CC BY) license (http://creativecommons.org/licenses/by/4.0/). 